\title{
THE STRUCTURE OF SOLAR ACTIVE REGIONS FROM EUV AND SOFT X-RAY OBSERVATIONS
}

\author{
CAROLE JORDAN* \\ Center for Astrophysics, Harvard College Observatory and Smithsonian Astrophysical \\ Observatory, Cambridge, Mass. 02138, U.S.A.
}

\begin{abstract}
The structure of solar active regions derived from EUV and soft X-ray observations is reviewed. The methods by which the emission measure as a function of temperature can be interpreted are discussed. The models of density and temperature which can be made from a variety of combinations of the emission measure with information on the spatial distribution of material, are broadly consistent. They show that the plasma at low heights over the central parts of an active region is hotter and denser than that which extends to greater heights. It appears that much of the emitting material exists in the form of loop structures, presumably magnetically controlled flux tubes. Analytical relationships between the physically important parameters describing the properties of the active region at $T_{\mathrm{e}}-2 \times 10^{5} \mathrm{~K}$ are developed and discussed.
\end{abstract}

\section{Introduction}

Active regions have been observed for many years at the levels of the photosphere and chromosphere, by making observations in the visible part of the spectrum. The literature on the visible region observations is extensive and good reviews can be found in Zirin (1966), Tandberg-Hanssen (1967), Kiepenheuer (1968) and Howard (1971).

In brief, active regions are characterized at photospheric levels by the presence of sunspots and concentrations of magnetic flux. In the chromosphere, lines such as $\mathbf{H} \alpha$ and $\mathrm{Ca} \mathrm{H}$ and $\mathrm{K}$ are strongly enhanced, and the definition of an active region includes such areas even in the absence of sunspots. Considerable fine structure, mostly of a filamentary nature is observed, for example, in $\mathrm{H} \alpha$. Because the magnetic field strengths are high these structures are often used to delineate the magnetic field configurations. In addition to the strong enhancement of emission over the areas surrounding the sunspots, features such as Arch Filament Systems (Bruzek, 1967, 1969) are observed extending into the corona when observations are made near the limb.

Before observations from rockets and satellites became possible the structure of the corona above active regions was studied from radio emission and from visible region photographs taken during eclipses. In addition to electron density determinations from the continuum intensities the temperature and density structure could be studied from the forbidden coronal lines formed between $\sim 10^{6}$ and $3 \times 10^{6} \mathrm{~K}$ (Aly et al., 1962). Since these observations could be made only at the limb little was known about the three-dimensional structure and usually cylindrical or hemispherical symmetry was assumed, in accordance with the general idea of a 'coronal condensation'. A useful summary of early radio studies of active regions can be found in the book by Kundu (1965).

* On leave of absence from the Appleton Laboratory, Astrophysics Research Division; Culham Laboratory, Abingdon, Berks., England. 
The EUV and X-ray observations provide the means of observing the active regions not only on the limb but on the disk, with a spatial resolution of $\sim 5^{\prime \prime}$. Since the spectrum includes lines which are formed at temperatures between $\sim 8000 \mathrm{~K}$ and $20 \times 10^{6} \mathrm{~K}$ (during flares), the whole structure from the chromosphere, through the transition region to the corona can be studied.

The present paper will review the models of the structure of active regions that have developed from the EUV and soft X-ray data.

\section{Studies of the Emission Measure}

\section{(a) FROM SOFT X-RAY SPECTRA}

Some of the early information on the hot components of active regions $\left(T_{\mathrm{e}}>2 \times 10^{6} \mathrm{~K}\right)$ came from soft $\mathrm{X}$-ray spectra of the whole sun at times when several active regions were present. Only recently have collimated instruments, which can allow spectra to be taken of individual active regions, been flown (Bonnelle et al., 1973; Brabban and Glencross, 1973). Unless high spatial resolution can be obtained, the interpretation of the emission line intensities is complicated by the presence of the emission from the whole quiet corona $\left(T_{\mathrm{e}} \sim 1.5 \times 10^{6} \mathrm{~K}\right)$. However, if uncollimated crystal spectrometers are used, the active regions present can appear as components to the observed spectral feature (e.g. See Batstone et al., 1970).

The initial step in analysing soft-X-ray spectra is to find the emission measure $\int_{\Delta V} N_{e}^{2} \mathrm{~d} V$ as a function of temperature. This can be done in the following way.

The emission in a particular line excited only by collisions from the ground level is given by

$$
E=8 \times 10^{-43} \bar{g} f \frac{N(E)}{N(\mathrm{H})} \int_{\Delta V} N_{e}^{2} g(T) \mathrm{d} V \operatorname{erg~cm}^{-2} \mathrm{~s}^{-1},
$$

where, following Pottasch (1963),

$$
g(T)=\frac{N(\text { ion })}{N(E)} T_{\mathrm{e}}^{-1 / 2} \exp \left(-W / k T_{\mathrm{e}}\right)
$$

$\bar{g}$ is the average Gaunt factor; $f$ is the oscillator strength; $N(E) / N(\mathrm{H})$ is the abundance of the element relative to hydrogen; $N($ ion $) / N(E)$ is the relative ion population in equilibrium conditions; $W$ is the excitation potential of the transition; $N_{\mathrm{e}}$ and $T_{\mathrm{e}}$ are the electron density and temperature respectively and $\Delta V$ is the volume of the atmosphere emitting a particular line.

The distribution of $N($ ion $) / N(E)$ and hence $g(T)$ with temperature can be calculated. A frequent approximation has been to replace $g(T)$ by a constant value over the region where most of the contribution to a given line occurs. Pottasch (1963) replaced $g(T)$ by $0.70 g\left(T_{\mathrm{m}}\right)$, where $g\left(T_{\mathrm{m}}\right)$ is the maximum value of $g(T)$ at $T_{\mathrm{m}}$. However, the temperature width to which this applies may differ from line to line, 
and to allow for this Jordan and Wilson (1971) replaced $g(T)$ by $G g\left(T_{\mathrm{m}}\right)$ such that

$$
G g\left(T_{\mathrm{m}}\right)=\int_{\log T_{1}}^{\log T_{2}} g(T) \mathrm{d} \log T
$$

and the normalization factor $G$ applies to a fixed temperature range $\Delta \log T=\log T_{\mathrm{m}}$ \pm 0.15 . $T_{1}$ and $T_{2}$ are chosen well outside the region of the maximum. With either method an average $g(T)$ can be removed from the integral and hence

$$
\frac{N(E)}{N(\mathrm{H})} \int_{\Delta V} N_{e}^{2} \mathrm{~d} V \quad \text { can be calculated for each line. }
$$

(The abundances may either be derived or assumed). The distribution of the emission measure $\int_{\Delta V} N_{\mathrm{e}}^{2} \mathrm{~d} V$ as a function of $T_{\mathrm{e}}$ is thus defined.

However, if the function $g(T)$ for a particular line does not vary steeply with $T_{\mathrm{e}}$, and the emission measure is varying rapidly with temperature, an iterative procedure must be adopted by recomputing $\int_{\Delta V} N_{\mathrm{e}}^{2} g(T) \mathrm{d} V$ until a self-consistent distribution is found. The temperature found for a given line depends strongly on the form of the emission measure, a fact which should be remembered if an empirical distribution is assumed for the emission measure. Also, if whole Sun observations are used it is important to take account of the large value of the emission measure at the temperature of the quiet corona.

Although this review is not primarily concerned with solar abundances it should be emphasized that the form of the emission function between $T_{\mathrm{e}} \sim 1.5 \times 10^{6} \mathrm{~K}$ and $6 \times 10^{6} \mathrm{~K}$ depends heavily on the abundances used and in particular authors differ in the abundance of neon that they use.

Beigman and Vainshtein (1970) analysed the whole Sun intensity data published by Evans and Pounds (1968), Walker and Rugge (1968) and Blake et al. (1965), by considering the emission as coming from a quiet coronal component and an active region component. They found that although the data of Walker and Rugge could be fitted by a two component model with little material between $T$ (corona) and $T$ (active), it was not possible for the other data to choose between such a model and one where the emission measure decreased smoothly with increasing $T_{\mathrm{e}}$.

Batstone et al. (1970) have used a least squares fit method to determine the variation of the emission measure with $T_{\mathrm{e}}$, for three active regions. Their results for one are shown in Figure 1, together with the values that could be obtained using the Pottasch type of analysis, before any iterations. It appears that a least squares method has little advantage over the Pottasch method plus iterations.

Chambe (1971) has also recognized that the $\int_{\Delta V} N_{\mathrm{e}}^{2} g(T) \mathrm{d} V$ should be calculated and has obtained a fit to various active region data by using an exponential form such that

$$
N_{\mathrm{e}}^{2} \frac{\mathrm{d} V}{\mathrm{~d} T}=C \times 10^{-T / T_{0}}
$$


where $T_{0}=1.5 \times 10^{6} \mathrm{~K}$ and $\mathrm{C}$ is a constant such that $N_{\mathrm{e}}^{2} \mathrm{~d} V / \mathrm{d} T=10^{49} \mathrm{~cm}^{-3} 10^{6} \mathrm{~K}^{-1}$ at $2 \times 10^{6} \mathrm{~K}$. His results are shown in Figure 2 . The reduction in the temperature at which $\mathrm{Ne}$ IX and $\mathrm{Ne} \mathrm{X}$ are formed is large; $T_{\mathrm{m}}$ for these ions has values of $3.5 \times 10^{6} \mathrm{~K}$ and $5.0 \times 10^{6} \mathrm{~K}$ respectively, but the temperatures at which most emission occurs are $2.4 \times 10^{6} \mathrm{~K}$ and $3.5 \times 10^{6} \mathrm{~K}$ respectively. Chambe's method as used by him did take account of the large emission measure at $T_{\mathrm{e}} \sim 1.5 \times 10^{6} \mathrm{~K}$, but the results obtained depend strongly on the exponential form assumed. In order to derive $N_{\mathrm{e}}$ and $T_{\mathrm{e}}$ as a function of height Chambe draws on a wide variety of visible region data, and it is

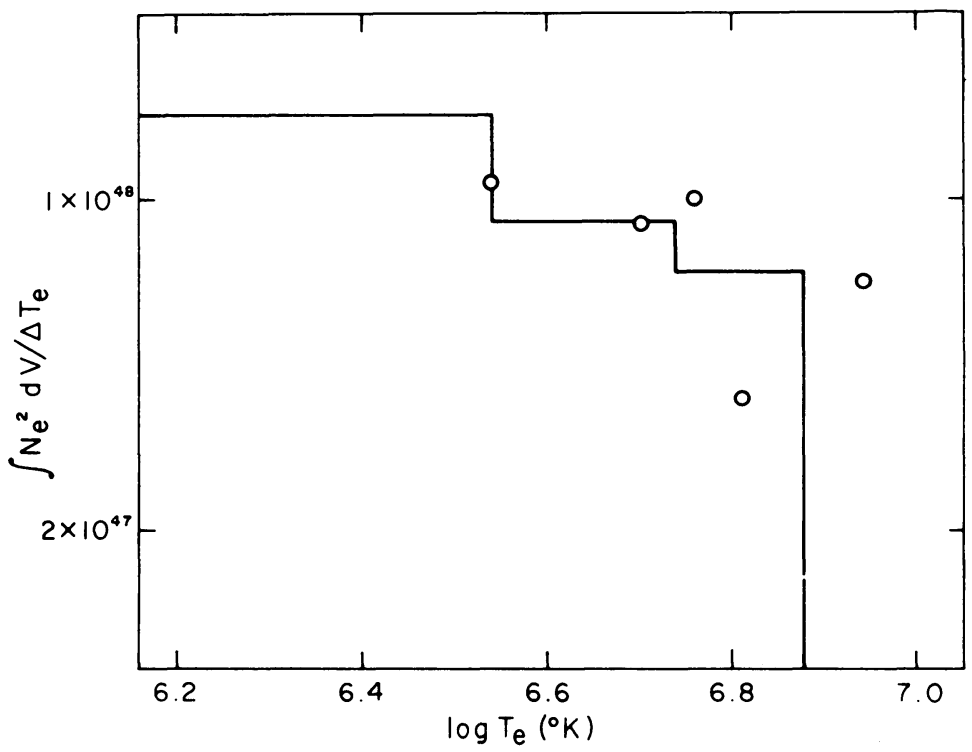

Fig. 1. Differential emission measure for an active region analysed by Batstone et al. (1970). The circles show the results of the Pottasch method of analysis.

difficult to realistically compare the significance of his results with others discussed in the present paper. From Figures 1 and 2 it can be seen that Chambe's function falls much more steeply as $T_{\mathrm{e}}$ increases than does the least squares fit.

Chambe's approach has been used by Acton et al. (1972) to analyse emission from $\mathrm{O}$ VII and Ne IX. Their spectra were obtained with a collimated instrument and emission from a $1.7^{\prime}$ wide strip was recorded. Although an exponential fit can be made between $\mathrm{O}$ VII and Ne IX, extrapolation back to lower temperatures is difficult since the emission measure at lower temperatures is not known for the individual active regions observed. Chambe's fit was to $\int_{\Delta V} N_{\mathrm{e}}^{2} \mathrm{~d} V$ for the whole corona plus any active region material present. The function used by Acton $e t$ al. continues to increase all the way down to $T_{\mathrm{e}}=10^{6} \mathrm{~K}$, and because of the steep variation of the emission measure with temperature the results will be affected by the assumed variation. This problem illustrates the importance of making observations of lines formed over a wide range of temperature. 


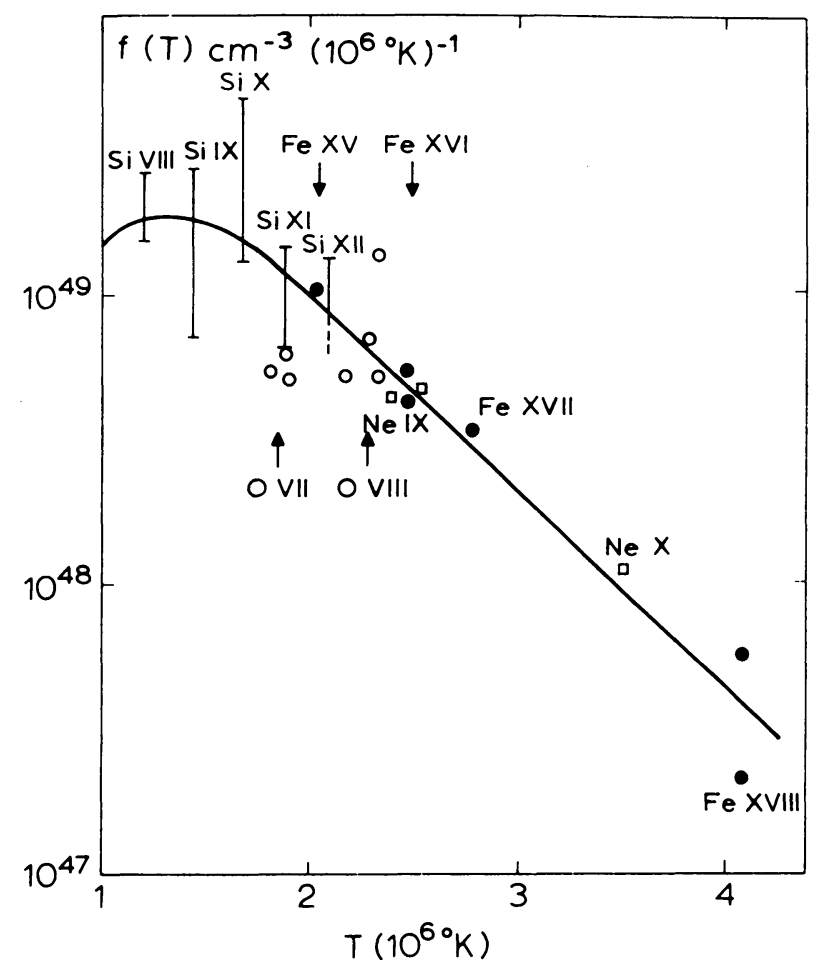

Fig. 2. Differential emission measure derived by Chambe (1971) using an exponential form (from Chambe, 1971).

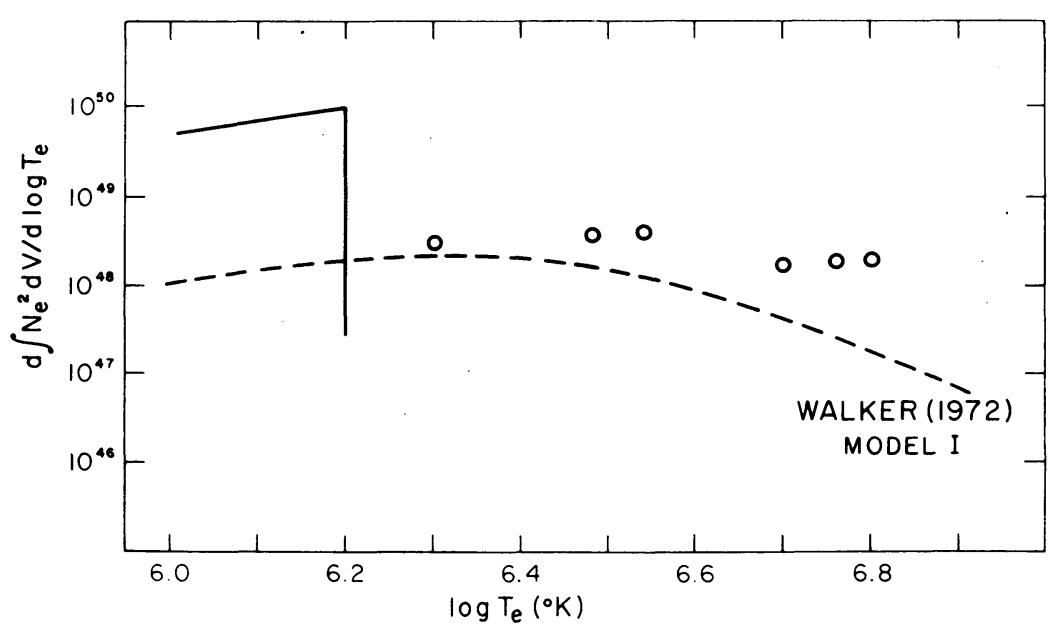

Fig. 3. Differential emission measure derived by Walker (1972), model I. The circles show the results of the Pottasch method of analysis. The full curve shows a schematic quiet coronal emission measure. 
Walker (1972) and Walker et al. (1973) have also used Chambe's approach, combining three exponential forms for $\int N_{\mathrm{e}}^{2} \mathrm{~d} V$ (for three temperature ranges) with a least squares fit to the data. These were whole Sun data, and the form of the emission function found by Pottasch (1967) was used for $10^{6} \mathrm{~K}<T<2 \times 10^{6} \mathrm{~K}$. Figure 3 shows Walker's Model I and also the results that would be obtained using $g(T)=0.70 g\left(T_{\mathrm{m}}\right)$, with no iteration. (Walker does not tabulate the atomic parameters he used; the values used in Figure 3 are $\bar{g}=0.30$ and $f$-values from Wiese et al. $(1966,1969))$. By using only the form of the emission function of the quiet corona between $10^{6}$ and $2 \times 10^{6} \mathrm{~K}$ Walker obtains a variation of the emission measure with temperature that is less steep than that of Chambe.

Walker et al. (1973) have analysed further data extending to higher temperature regions using the same method and with similar results. Also Bonnelle et al. (1973) have recently analysed their collimated $\mathrm{Mg}$ XI and $\mathrm{Mg}$ XII data by fitting an exponential fall-off curve and find results consistent with those of other authors.

It is possible to make emission measure studies even from broad band X-ray flux measurements. Landini and Monsignori Fossi (1971) have combined observations made with the Solrad 9 satellite with their theoretical calculations of the temperature dependence of emission integrated over large wavelength bands and have derived a combined emission measure between $10^{6}$ and $3 \times 10^{7} \mathrm{~K}$.

Although the observed distribution of the emission measure can be fitted by functional forms it is not possible to separate $\int_{\Delta V} N_{\mathrm{e}}^{2} \mathrm{~d} V$ into density, temperature gradient and volume components without further assumptions or information on the spatial distribution of the emission.

\section{(b) FROM EUV SPECTROHELIOGRAMS AND SPECTRA}

The Harvard College Observatory instruments on OSO 4 and OSO 6 have provided observations of the structure of active regions over the temperature range $10^{4}-3.5 \times 10^{6} \mathrm{~K}$. Noyes (1971) has reviewed analyses of data from both these experiments. The OSO 4-observations were in the form of whole Sun raster spectroheliograms, made over an interval of $5 \mathrm{~min}$, in selected emission lines. The spatial resolution was $1^{\prime}$ square. The interpretation of these observations has been discussed by Noyes et al. (1970). The main parameter studied was the enhancement in an active region of a given line over its average quiet Sun value. Figure 4 shows the average results from eight active regions. It can be seen that the enhancements vary from about a factor of 3 for transition region ions up to about 40 for Si XII, the highest ion plotted. The enhancement varies from line to line at a given temperature because of differing density dependences, an immediate indication that the density has changed between the quiet and active regions.

The density dependence of the lines of Be I-like ions, in particular of C III, N IV and $\mathrm{O} \mathrm{v}$ can in principle be used to determine the electron density in the quiet and active regions, (Jordan, 1971a; Munro et al., 1971), but at present the method is limited by uncertainties in the necessary atomic data (Jordan, 1974). However, the data reported by Noyes et al. show that the density enhancements for $\mathrm{C}$ III and $\mathrm{O} \mathrm{v}$ are between 


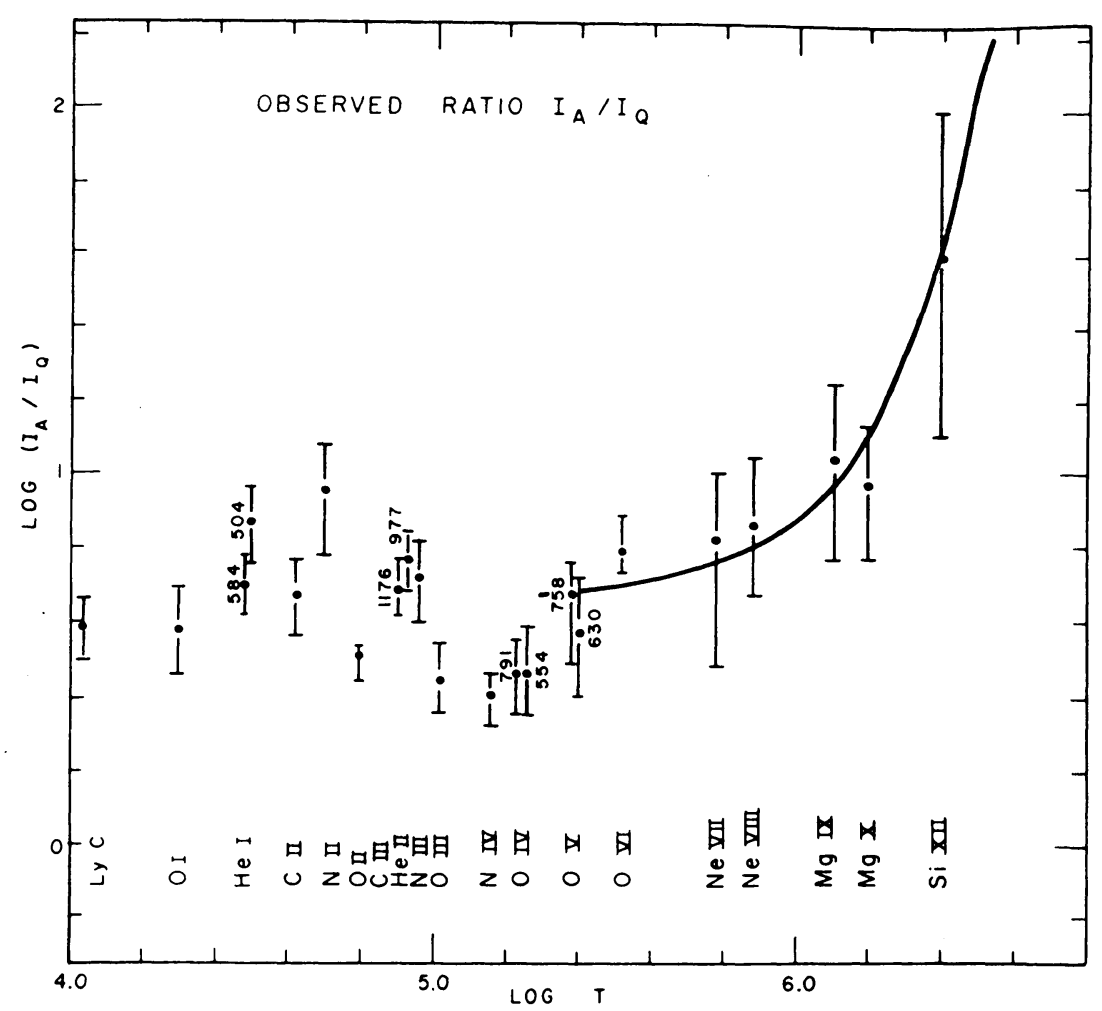

Fig. 4. Relative intensities of lines between quiet and active regions observed from OSO 4. (From Noyes et al., 1970).

factors of $\sim 2$ to 6 . Since independent measures of the density allow other parameters such as the conductive flux $F_{\mathrm{c}}$, and the temperature gradient to be determined, the method is potentially extremely valuable.

The analysis will now be treated in a more general way than used by Withbroe (1970). From spectra of the quiet sun it has been shown that between $T_{\mathrm{e}} \sim 10^{5}$ to $10^{6} \mathrm{~K}$ the emission measure has a gradient which is close to that expected if there was constant conductive flux back from the corona (Athay, 1966). This can be seen from the following. The observed emission measure shows that:

$$
\int_{\Delta T} N_{\mathrm{e}}^{2} \frac{\mathrm{d} h}{\mathrm{~d} T} \mathrm{~d} T=a T^{b} .
$$

The conductive flux is

$$
F_{\mathrm{c}}=k T^{5 / 2} \mathrm{~d} T / \mathrm{d} h \mathrm{erg} \mathrm{cm}^{-2} \mathrm{~s}^{-1}
$$

Using $P_{\mathrm{e}}=N_{\mathrm{e}} T_{\mathrm{e}}$, Equations (3) and (4) give the relation

$$
F_{\mathrm{c}}={ }_{a b}^{k P_{\mathrm{e}}^{2}} T^{-b+3 / 2}
$$


assuming also that the emission is from a spherically symmetric atmosphere. A condition for $F_{\mathrm{c}}$ to be constant is then $b=\frac{3}{2}$ and $P_{\mathrm{e}}=$ const. The regime over which $P_{\mathrm{e}}$ can be assumed to be constant can be examined by using the additional hydrostatic equilibrium relation that:

Then

$$
\frac{\mathrm{d} \log P_{e}}{\mathrm{~d} h}=-0.86 \times 10^{-4} \frac{1}{T_{e}}
$$

$$
P_{\mathrm{e}}^{2}=P_{0}^{2}-\frac{D a}{(b+1)}\left(T_{\mathrm{e}}^{b+1}-T_{0}^{b+1}\right),
$$

where $D=4 \times 10^{-4} b$.

If $b=\frac{3}{2}$, then using a typical quiet region value for $\int N_{\mathrm{e}}^{2} \mathrm{dh}$ at $2 \times 10^{5} \mathrm{~K}$ (Dupree, 1972), gives $a=3.3 \times 10^{17}$. If $P_{0}=6 \times 10^{14} \mathrm{~cm}^{-3} \mathrm{~K}$, and $T_{0}=2 \times 10^{5} \mathrm{~K}$, the condition on the temperature range over which $P_{\mathrm{e}}$ can be taken as constant (i.e. $P_{\mathrm{e}}^{2}=P_{0}^{2}$ to within $10 \%$ ) is $T_{0}<T_{\mathrm{e}}<7 \times 10^{5} \mathrm{~K}$. If $T_{\mathrm{e}} \gg T_{0}$, then

$$
P_{\mathrm{e}}^{2}=P_{0}^{2}-\frac{D a}{(b+1)} T_{\mathrm{e}}^{5 / 2}
$$

and if the temperature of the isothermal corona above the transition region is taken as the value where $P_{\mathrm{e}} \rightarrow 0$, then $T_{\mathrm{c}} \rightarrow 1.8 \times 10^{6} \mathrm{~K}$. The atmosphere must have sufficient height for its temperature to approach this limit. This determination of $T_{\mathrm{c}}$ allows $F_{\mathrm{c}}$ at $P_{0}$ to be found directly if $T_{\mathrm{c}}$ is known, since

$$
T_{\mathrm{c}}^{5 / 2}=\left(\begin{array}{c}
P_{0}^{2} \\
a
\end{array}\right) \cdot\left(\begin{array}{c}
b+1 \\
D
\end{array}\right)
$$

and

$$
F_{\mathrm{c}}\left(P_{0}\right)=\frac{k P_{0}^{2}}{a} \frac{1}{b}
$$

Thus

$$
F_{\mathrm{c}}\left(P_{0}\right)=\frac{k}{b} \cdot \frac{D}{(b+1)} \cdot T_{\mathrm{c}}^{5 / 2} .
$$

Although not expressed in this way the above relations are the physical basis behind Withbroe's (1970) empirical three-parameter description of the structure of the quiet atmosphere. He describes models in terms of $F_{\mathrm{c}}, P_{0}$ and $T_{\mathrm{c}}$. His method has been used by Noyes et al. (1970) to investigate the changes in $P_{0}, F_{\mathrm{c}}$ and $T_{\mathrm{c}}$ between the quiet and active regions. From the distribution shown in Figure 4 they deduce that to account for the observed variation of both transition region and coronal lines requires an increase in $P_{0}$ and $F_{\mathrm{c}}$ of a factor of five, and an increase of $T_{\mathrm{c}}$ to $2.5 \times 10^{6} \mathrm{~K}$.

These results are consistent with the analytical method using the increased value of the constant ' $a$ ' given by the observed enhancement of a factor of $\simeq 5$ at $T_{\mathrm{e}}=T_{0}=$ $=2 \times 10^{5} \mathrm{~K}$, and a density enhancement of a factor of 5 determined from the $\mathrm{O} v$ ratio. 
If $N_{\mathrm{e}}$ increases by a factor of 5 at $T_{0}$, then $\left(P_{0}\right)$ active $=5\left(P_{0}\right)$ quiet and $\left(F_{\mathrm{c}}\right)$ active $=5\left(F_{\mathrm{c}}\right)$ quiet. Using Equation (7), the regime where $P_{\mathrm{e}}=$ constant now extends to $T_{\mathrm{e}}=9.6 \times 10^{5} \mathrm{~K}$, and it is found that $T_{\mathrm{c}}=2.4 \times 10^{6} \mathrm{~K}$.

Both spectra and spectroheliograms were obtained with the OSO 6 instrument. The spatial resolution for regions where spectra were obtained was $35^{\prime \prime}$. In an analysis of McMath Region 10266 Dupree et al. (1973) use Withbroe's arguments and the O v ratio to show that $P_{0}$ (active) $/ P_{0}$ (quiet) $=7$, that $F_{\mathrm{c}}=1.5 \times 10^{7} \mathrm{~cm}^{-2} \mathrm{~s}^{-1}$ (a factor of 12 greater than the quiet value) and $T_{\mathrm{c}}=3.2 \times 10^{6} \mathrm{~K}$. Proceeding analytically, the density increase of a factor of 7 can be combined with an intensity increase of 5 , to say that $F_{\mathrm{c}}$ increases by a factor of 10 , and $T_{\mathrm{c}}=3.2 \times 10^{6} \mathrm{~K}$.

Thus by measuring both $N_{\mathrm{e}}$ and the intensity enhancement at $2 \times 10^{5} \mathrm{~K}$, or by determining $T_{\mathrm{c}}$ and $N_{\mathrm{e}}$ the structure of the active region at $T_{\mathrm{e}}>2 \times 10^{5} \mathrm{~K}$ (treated as an average) can be calculated.

\section{(c) STUDIES OF LIMB SPECTRA}

Limb observations have the advantage that the variation of the emission measure in lines formed at different temperatures can be studied as a function of height. Prior to the recent Skylab observations active regions observed on spectroheliograms on the disk were usually treated as single features.

The EUV spectroheliograms obtained by the Naval Research Laboratory group can be used to study the relative spatial distribution of material as a function of temperature (See e.g. Tousey, 1967). Boardman and Billings (1969) have made an analysis of an active region seen on the limb during an Aerobee 150 rocket flight by the NRL group on April 28, 1966. They use a Pottasch type of analysis, and from the intensities of a variety of lines they deduce the emission measure as a function of temperature and position. By assuming a cylindrical symmetry about the central point of the region they derive $\left\langle N_{\mathrm{e}}^{2}\right\rangle^{1 / 2}$ as a function of temperature and height. In view of the loop structures observed from the X-ray photographs (see Section III(a)) the cylindrical approximation may not be appropriate. However, the overall results that they obtain (shown in Figure 5), do not differ substantially from those obtained by Gabriel and Jordan (1974) (see below), if the spatial resolution of the latter results was reduced. Boardman and Billings find that the region has a dense hot 'core' of about $20000 \mathrm{~km}$ in height and $30000 \mathrm{~km}$ radius, which is surrounded by cooler, less dense material. The temperature ranges from $3 \times 10^{6} \mathrm{~K}$ in the core down to $1.7 \times 10^{6} \mathrm{~K}$ at $4 \times 10^{4} \mathrm{~km}$. The density falls from $2 \times 10^{9} \mathrm{~cm}^{-3}$ in the core to $1.3 \times 10^{8} \mathrm{~cm}^{-3}$ at $4 \times 10^{4} \mathrm{~km}$.

During the 1970, March 7 total eclipse a rocket was launched from Wallops Island, Virginia into the eclipse path. This experiment and the data obtained have been described by Speer et al. (1970) and by Gabriel et al. (1971). Some thirty five photographic slitless spectra were obtained over the spectral range $850 \AA$ to $2190 \AA$ during the flight with a grain-limited spatial resolution of about 3 ". One of the interesting features observed was a large loop system and intensity enhancement on the north-east limb. From the Fraunhofer Institute maps and the magnetograms of Livingston et al. (1970) 
this was probably associated with McMath 10623, which was a weak plage region with no sunspots recorded on the maps. The coronal component of the active region is apparent in the forbidden lines of silicon, iron, sulphur and nickel which lie between $1000 \AA$ and $2200 \AA$, and which were first identified from this data (Gabriel et al., 1971; Jordan, 1971b). The active region can be seen in lines formed at temperatures between

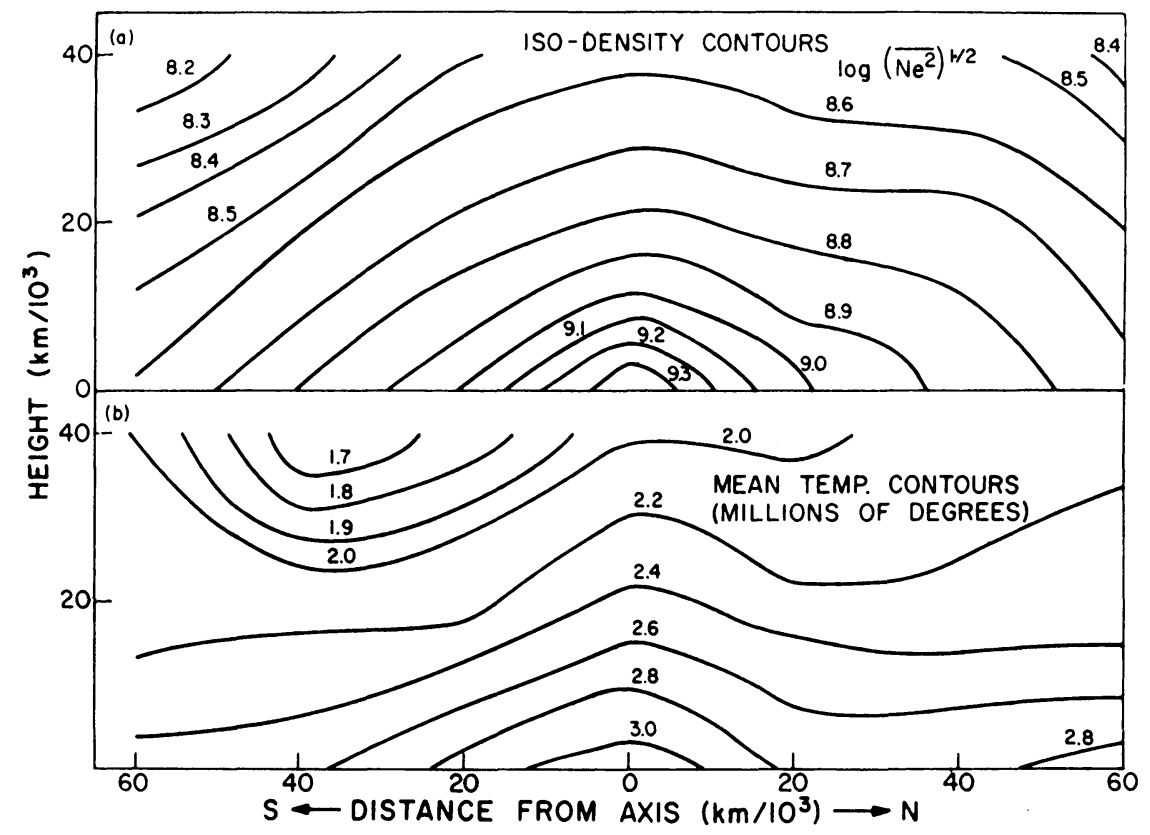

Fig. 5. The density and temperature as a function of position in a limb active region observed by the Naval Research Laboratory (from Boardman and Billings, 1969).

$7 \times 10^{5} \mathrm{~K}$ and $2.5 \times 10^{6} \mathrm{~K}$, and the apparent change in the structure as a function of temperature is shown in Figure 6.

Gabriel and Jordan (1974) have recently made an analysis of the density and temperature structure as a function of height and position in the active region. The spectra were intensity calibrated so that analyses of both absolute and relative intensities have been possible. Frame 30, taken when the Moon's limb was $10950 \mathrm{~km}$ above the photosphere, at the latitude of the active region, has been used in the analysis, although a normalization procedure for level populations was made using quiet coronal data over a series of frames.

The method by which the emission measure may be derived has been given in Section II(a). For forbidden lines the approximation that the excited level is populated mainly from the ground is not always valid, so instead of replacing $N_{2} A_{21}$ by $C_{12} N_{\mathrm{e}} N_{1}$, as was done in deriving Equation (1), the upper level population is retained and can be calculated as a function of $N_{\mathrm{e}}$ and $T_{\mathrm{e}} . N_{1}$ and $N_{2}$ are the populations of the ground and upper levels respectively, $A_{21}$ is the spontaneous transition probability, $C_{12}$ is the 


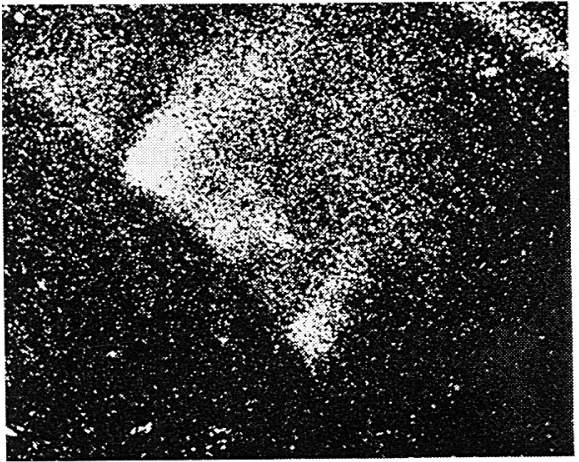

Si vIII. $1446 \AA . \quad\left(9.3 \times 10^{5} \circ\right.$ K.)

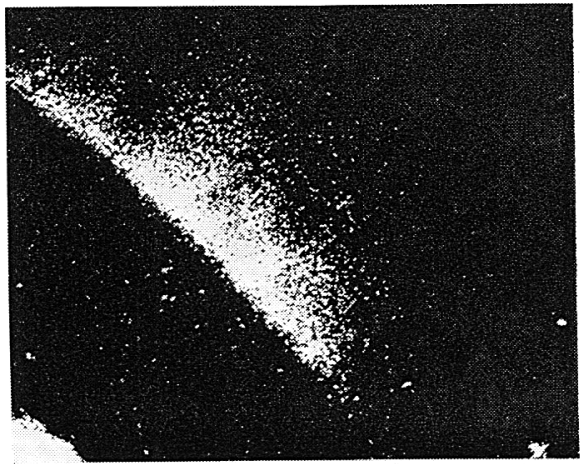

Fe $x 11 . \quad 1350$ \&. $\quad\left(1.7 \times 10^{6} \cdot k.\right)$

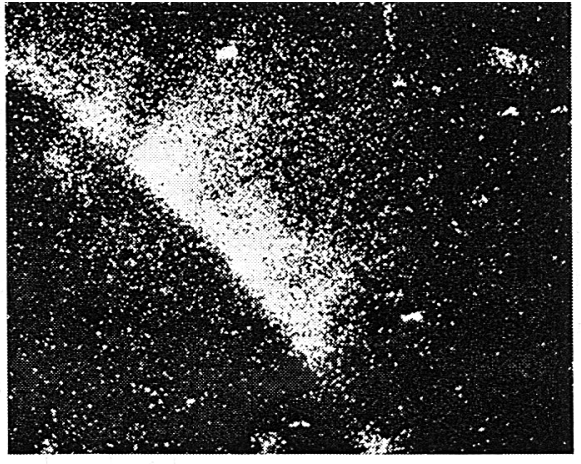

Fe $X 1 . \quad 1467 \AA . \quad\left(1.5 \times 10^{6} \cdot \mathrm{K}.\right)$

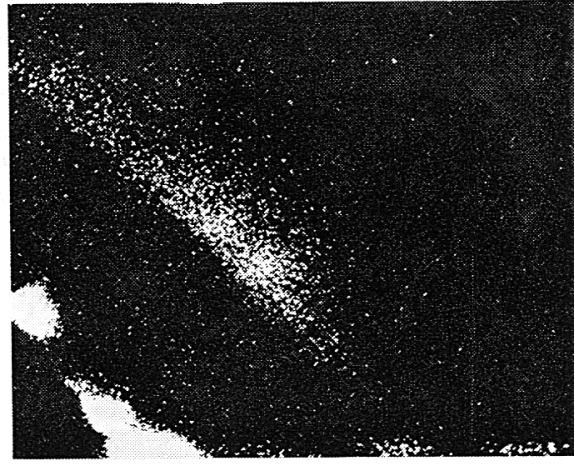

$5 \times 1 . \quad 1826 \pi . \quad\left(2.0 \times 10^{6} \mathrm{~K}.\right)$

Fig. 6. A limb active region observed in forbidden lines of Si VIII, Fe XI, Fe XII and S XI during the March 1970 total eclipse (from Gabriel et al., 1971).

collisional excitation rate. Hence at a given point in the active region

$$
I=\text { const. } \begin{aligned}
& N(E) \\
& N(\mathrm{H})
\end{aligned} \int_{\Delta L} \frac{N_{2}}{N(\text { ion })} \cdot \frac{N(\text { ion })}{N(E)} \cdot N_{\mathrm{e}} \mathrm{d} l \quad \mathrm{erg} \mathrm{cm}^{-2} \mathrm{~s}^{-1} \mathrm{sterad}^{-1}
$$

where the integration is now over $\Delta L$, the line of sight path length for a particular line.

$N_{2} / N$ (ion) can be calculated as a function of $N_{\mathrm{e}}$ and $T_{\mathrm{e}}$, and for the lines of Si IX, Fe XI, Fe XII $\left({ }^{4} S-{ }^{2} P\right)$ and $\mathrm{S} \mathrm{XI}, N_{2} / N($ ion $) N_{\mathrm{e}} \simeq$ constant, and $I \propto N_{\mathrm{e}}^{2}$, as for many permitted resonance lines. For the lines of Si VIII and Fe XII $\left({ }^{4} S-{ }^{2} D\right)$ a density must be assumed initially and iterations performed as necessary. The values of $N($ ion $) / N(E)$ have been taken from the calculations by Jordan (1969).

Thus

$$
I=\text { const. }\left(\begin{array}{cc}
N_{2} & 1 \\
N_{\text {ion }} & N_{\mathrm{e}}
\end{array}\right) \int_{\Delta L} N_{\mathrm{e}}^{2} \mathrm{~d} l,
$$

and the emission measure $\int_{\Delta L} N_{\mathrm{e}}^{2} \mathrm{~d} l$ can be found. 
The details of the atomic parameters used are given by Gabriel and Jordan, and only the resulting structure found by them will be discussed at present.

Figure 7 shows the distribution of $\int_{\Delta L} N_{\mathrm{e}}^{2} \mathrm{~d} l$ as a function of temperature and position in the active region. (The loops apparent in Si vIII are sketched in to aid the location of the position in Figure 6). Overall the results shown in Figure 7 confirm the impression, from Figure 6, that the central area contains more hot material than does the loop system apparent in Si viII, and that the fraction of hot material present decreases with increasing height.

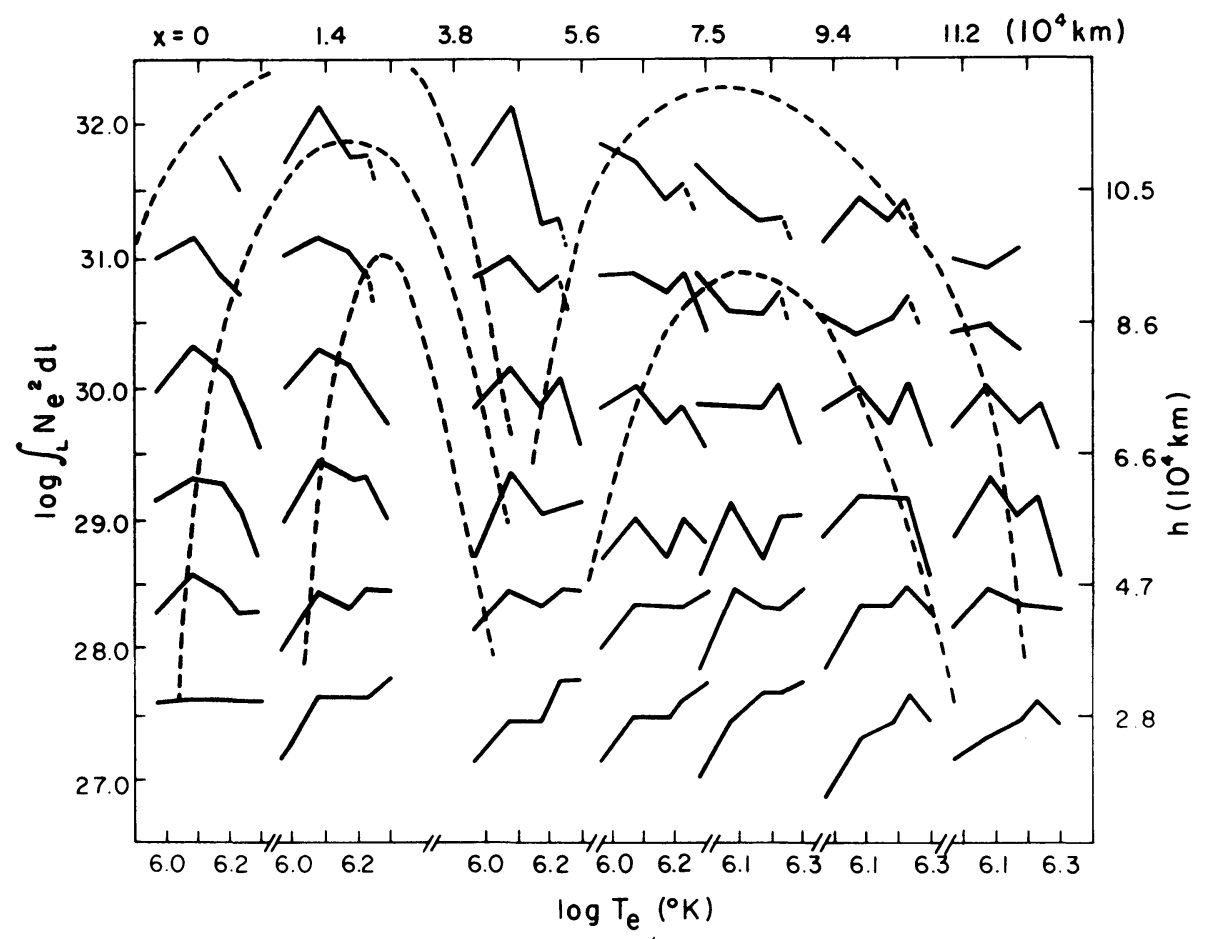

Fig. 7. The emission measure $\int \Delta_{L} N_{\mathrm{e}}^{2} \mathrm{~d} l$ as a function of position in the 1970, March 7 limb active region. $x$ is the distance from the left side of the region, $h$ is the height above the photosphere.

In order to resolve $\int_{\Delta L} N_{\mathrm{e}}^{2} \mathrm{~d} l$ into components of $N_{\mathrm{e}}$ and $\Delta L$ for each line, some independent measure of either $N_{\mathrm{e}}$ or $\Delta L$ is necessary. Three methods can be used. Assuming that the loop structures are similar in shape to those seen by Van Speybroeck et al. (1970) it can be assumed that $\Delta L=d$, the observed diameter of the loop. This gives an upper limit to $\Delta L$ for the loop may not be filled with the relevant material. However, at $28000 \mathrm{~km}$, (column 1 in Figure 7), $\Delta L=3.6 \times 10^{4} \mathrm{~km}$ leads to $N_{\mathrm{e}}=1.0 \times 10^{9} \mathrm{~cm}^{-3}$ at $T_{\mathrm{e}}=9.3 \times 10^{5} \mathrm{~K}\left(N_{\mathrm{e}} T_{\mathrm{e}}=9.3 \times 10^{14} \mathrm{~cm}^{-3}\right)$. Alternatively, since the Si vill emission depends on $N_{\mathrm{e}}$ rather than $N_{\mathrm{e}}^{2}$ over the density range $10^{9}-10^{10} \mathrm{~cm}^{-3}$ 
an average density can be found from the relative intensities of the Si VIII and $\mathrm{Si}$ IX lines. This is only a crude method since it assumes that $N_{\mathrm{e}}$ and $\Delta L$ are the same for Si vill and Si IX. However, the method gives the results shown in Table I. The positions correspond to the first, fourth and seventh columns in Figure 7. Densities of $N_{\mathrm{e}} \sim 1-2 \times 10^{9} \mathrm{~cm}^{-3}$ are found with $\Delta L$ between $3 \times 10^{3}$ and $3 \times 10^{4} \mathrm{~km}$. The fact that $N_{\mathrm{e}}$ does not fall by the value expected in hydrostatic equilibrium with $T_{\mathrm{c}}=$ const. - contrary to the results of analyzing the decrease in the absolute intensity with height, presented below - is probably due to the crudeness of the method.

\section{TABLE I}

$N_{\mathrm{e}}$ and $\Delta L$ from $\mathrm{Si}$ VIII/Si Ix intensity ratios in 1970, March 7 eclipse active region

\begin{tabular}{|c|c|c|c|c|c|c|}
\hline \multirow{2}{*}{$\begin{array}{l}\text { Height } \\
(\mathrm{km})\end{array}$} & \multicolumn{2}{|c|}{ Region A } & \multicolumn{2}{|c|}{ Region B } & \multicolumn{2}{|c|}{ Region C } \\
\hline & $\begin{array}{l}\log N_{\mathrm{e}} \\
\left(\mathrm{cm}^{-3}\right)\end{array}$ & $\begin{array}{l}\Delta L \\
10^{4} \mathrm{~km}\end{array}$ & $\begin{array}{l}\log N_{\mathrm{e}} \\
\left(\mathrm{cm}^{-3}\right)\end{array}$ & $\begin{array}{l}\Delta L \\
10^{4} \mathrm{~km}\end{array}$ & $\begin{array}{l}\log N_{\mathrm{e}} \\
\left(\mathrm{cm}^{-3}\right)\end{array}$ & $\begin{array}{l}\Delta L \\
10^{4} \mathrm{~km}\end{array}$ \\
\hline 30000 & 9.02 & 3.5 & 9.12 & 1.2 & 8.98 & 1.9 \\
\hline 49000 & 9.17 & 1.3 & 9.33 & 0.45 & 9.28 & 0.68 \\
\hline 67000 & 9.20 & 0.81 & 9.25 & 0.32 & 9.40 & 0.26 \\
\hline 86000 & 9.16 & 0.74 & 9.10 & 0.51 & 9.04 & 0.32 \\
\hline 105000 & 9.05 & 1.1 & 9.05 & 0.57 & 8.97 & 0.30 \\
\hline
\end{tabular}

A third method of determining $N_{\mathrm{e}}$ is to use the relative intensities of the Fe XII lines ${ }^{4} S_{3 / 2}-{ }^{2} D_{3 / 2}$ and ${ }^{4} S_{3 / 2}-{ }^{2} P_{1 / 2}$ which are sensitive to $N_{\mathrm{e}}$ over the range $10^{12}>N_{\mathrm{e}}>10^{8} \mathrm{~cm}^{-3}$. The results are given in Table II. The density, now at $T_{\mathrm{e}}=1.7 \times 10^{6} \mathrm{~K}$, falls from $N_{\mathrm{e}}=3 \times 10^{9} \mathrm{~cm}^{-3}$ at $30000 \mathrm{~km}$ to $\sim 10^{9} \mathrm{~cm}^{-3}$ by $10^{5} \mathrm{~km}$. In hydrostatic equilibrium with $T_{\mathrm{e}}=1.7 \times 10^{6} \mathrm{~K}$, a fall of a factor of 2-3 would be expected. The path length from the Fe XII emission varies between $2 \times 10^{3} \mathrm{~km}$ to $1.5 \times 10^{5} \mathrm{~km}$, but on the whole does not increase substantially with height. However, the path length derived at $1.7 \times 10^{6} \mathrm{~K}$ is systematically lower than that derived from the Si VIII/Si IX ratio.

TABLE II

Electron densities and $\Delta L$ from Fe XII ratios in 1970, March 7 eclipse active regions

\begin{tabular}{lllllll}
$\begin{array}{l}\text { Height } \\
10^{4}(\mathrm{~km})\end{array}$ & \multicolumn{2}{c}{ Region A } & \multicolumn{3}{c}{ Region B } & \multicolumn{2}{c}{ Region C } \\
\cline { 2 - 7 } & $\begin{array}{l}\log N_{\mathrm{e}} \\
\left(\mathrm{cm}^{-3}\right)\end{array}$ & $\begin{array}{l}\Delta L \\
10^{3} \mathrm{~km}\end{array}$ & $\begin{array}{l}\log N_{\mathrm{e}} \\
\left(\mathrm{cm}^{-3}\right)\end{array}$ & $\begin{array}{l}\Delta L \\
10^{3} \mathrm{~km}\end{array}$ & $\begin{array}{l}\log N_{\mathrm{e}} \\
\left(\mathrm{cm}^{-3}\right)\end{array}$ & $\begin{array}{l}\Delta L \\
10^{3} \mathrm{~km}\end{array}$ \\
3.0 & 9.58 & 2.7 & 9.56 & 4.3 & 9.43 & 3.9 \\
4.9 & 9.45 & 3.5 & 9.42 & 4.1 & 8.99 & 14.8 \\
6.7 & 9.44 & 2.6 & 9.25 & 4.5 & 9.12 & 5.8 \\
8.6 & 9.36 & 2.7 & 9.27 & 2.9 & 9.22 & 2.6 \\
10.5 & 9.11 & 4.3 & 9.15 & 5.0 & &
\end{tabular}


The results for two points at $30000 \mathrm{~km}$ (column 1 and column 4 in Figure 7) are summarized in Table III. The pressure increases from $\sim 10^{15} \mathrm{~cm}^{-3} \mathrm{~K}$ at $9 \times 10^{5} \mathrm{~K}$ to $6 \times 10^{15} \mathrm{~cm}^{-3} \mathrm{~K}$ at $2 \times 10^{6} \mathrm{~K}$. (The typical quiet transition region value is $6 \times$ $\times 10^{14} \mathrm{~cm}^{-3} \mathrm{~K}$, and for the quiet corona is $\simeq 4 \times 10^{14} \mathrm{~cm}^{-3} \mathrm{~K}$ ). Only small magnetic fields would be necessary to balance the gas pressure - between 2 and $5 \mathrm{G}$. The values of $N_{\mathrm{e}}$ and $\Delta L$ have been interpolated between the values for Si vIII and Fe XII.

TABLE III

Models for selected parts of 1970, March 7 eclipse active region

Model for Region A at $h=30000 \mathrm{~km}$

$\begin{array}{llll}\log T & \text { Ion } & \log N_{\mathrm{e}} & \Delta L(\mathrm{~km}) \\ 5.97 & \text { Si VIII } & 9.02 & 3.5 \times 10^{4} \\ 6.08 & \text { Si IX } & 9.24 & 1.3 \times 10^{4} \\ 6.18 & \text { Fe XI } & 9.44 & 5.0 \times 10^{3} \\ 6.23 & \text { Fe XII } & 9.58 & 2.7 \times 10^{3}\end{array}$

Model for Region B at $h=30000 \mathrm{~km}$

$\begin{array}{llll}5.97 & \text { Si VIII } & 9.12 & 1.2 \times 10^{4} \\ 6.08 & \text { Si IX } & 9.30 & 6.2 \times 10^{3} \\ 6.18 & \text { Fe XI } & 9.46 & 3.1 \times 10^{3} \\ 6.23 & \text { Fe XII } & 9.56 & 4.3 \times 10^{3}\end{array}$

It remains to determine how the material in a given line of sight is distributed in position. Is the hot material within the cool or vice versa? If the active region is considered as composed of adjacent flux tubes, and the hot material is within the cool, the path length for the cool material is sufficiently large for 'gaps' to appear between adjacent regions emitting high temperature lines. This is not observed. At $30000 \mathrm{~km}$ the apparent distribution is consistent with a set of loops each with a structure such that the cool material is within the hot. The fraction of the loop material at high temperatures increases towards the central regions. Small-scale helical structures within the loop systems are not ruled out by the observations.

A further study that has been made by Gabriel and Jordan is of the decrease in the observed emission as a function of height. For lines which have intensity $\propto N_{\mathrm{e}}^{2}$ it would be expected that in the absence of a magnetic field - or along a field line, that the emission per unit volume would eventually decrease according to Equation (6) for hydrostatic equilibrium. The calculated $N_{\mathrm{e}}$ dependence starting from a base density can also be combined with Equation (6) if the line does not have intensity $\propto N_{\mathrm{e}}^{2}$. If the line of sight distance $\Delta L$ increased as a function of height, as might be expected with the expansion of a dipole type field, then this would show as a departure from a hydrostatic fall off with constant $T_{\mathrm{e}}$. Figure 8 shows the intensity (on a relative scale) 
observed as a function of height in Si VIII, Si IX and Fe XI, following as well as possible the 'field' direction in the left-hand loop of Figure 7. The hydrostatic fall-off with $T_{\mathrm{e}}=T_{\mathrm{m}}$ for each ion, and the density corrected curves are also given. It can be seen that for heights above $40000 \mathrm{~km}$, the hydrostatic curve is closely followed by the Si IX and Fe XI emission. An increase in $\Delta L$ could raise the curve, but a decrease in $T_{\mathrm{e}}$ with height would lower the curve. Unless these two effects cancel out fairly precisely,

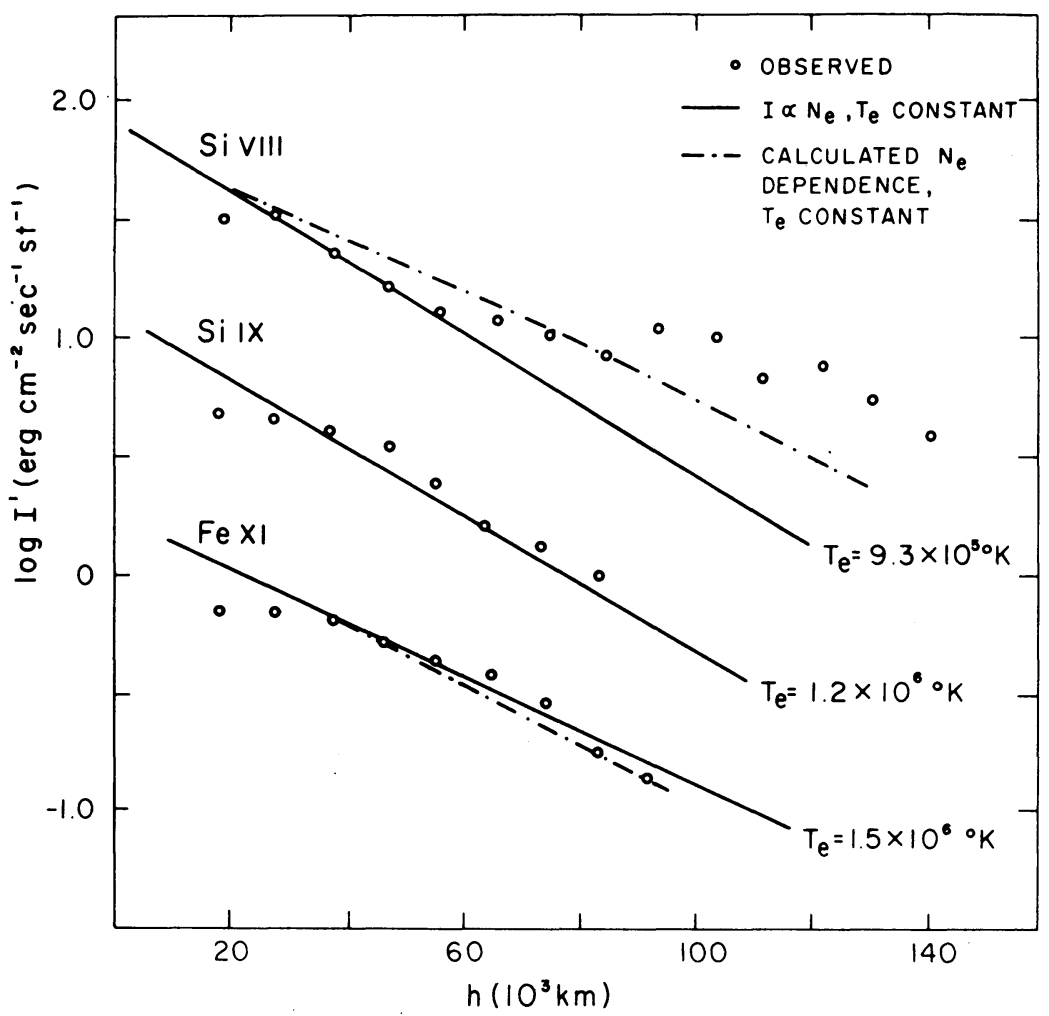

Fig. 8. The decrease of emission with height in the Si vII, Si IX and Fe XI, forbidden lines on the left side of the loop structure observed in the 1970, March 7 limb active region. The full lines show the decrease expected in hydrostatic equilibrium at $T_{m}$, if the emission is proportional to $N_{\mathrm{e}}$. The dashed lines take into account the calculated dependence on $N_{\mathrm{e}}$. The intensities, $I^{\prime}$, are on relative scales.

Figure 8 shows that $\Delta L$ is practically constant with height up to $\sim 9 \times 10^{5} \mathrm{~km}$. The Si vill emission above $9 \times 10^{5} \mathrm{~km}$ shows that $\Delta L$ eventually increases by a factor of about 2. The values of $\Delta L$ can be combined with the emission measures to find $N_{\mathrm{e}}$ as a function of height. There appears to be a systematic departure from the straight line for emission at $h<4.0 \times 10^{4} \mathrm{~km}$. This could be interpreted as evidence for a nonisothermal medium - i.e., individual loops no longer have a basically isothermal structure. Studies of the transition region material at heights up to $40000 \mathrm{~km}$ should allow the type of analysis outlined in the previous section to be made for this active region. 


\section{The Spatial Distribution of Material in Active Regions}

\section{(a) FROM X-RAY IMAGES}

The spatial resolution obtainable in images of X-ray emitting regions is now $\sim 2$ ". The early photographs obtained at 20" resolution (Underwood and Muney, 1967), showed how the material with $T_{\mathrm{e}} \geqslant 2 \times 10^{6} \mathrm{~K}$ is concentrated over active regions. By making observations through filters which had different responses as a function of wavelength Underwood and Muney found that the radiation at longer wavelengths (lower $T_{\mathrm{e}}$ ) was more diffuse in origin than that at shorter wavelengths. With improved resolution it became possible to observe some of the structure present in the X-ray emitting regions. The photographs obtained by the American Science and Engineering (AS \& E) Group (Krieger et al., 1971, and earlier papers) have shown that the material at $T_{\mathrm{e}}>2 \times 10^{6} \mathrm{~K}$ is characteristically in the form of loop structures, connecting regions of opposite polarity observed with ground-based magnetographs. The loops have similarities to those seen earlier in white light photographs but do not coincide with $\mathrm{H} \alpha$ structures. The outline of the X-ray emission follows in general that of the underlying $\mathrm{Ca} \mathrm{K}$ plage, and is associated with enhanced magnetic fields. The loops have a range of sizes and frequently reach heights of $\sim 1.5 \times 10^{5} \mathrm{~km}$ above the photosphere. Figure 9 shows the photograph obtained by Van Speybroeck et al. (1970) just after the 1970, March 7 solar eclipse. The form of the loops strongly suggests that the emitting material is confined by the magnetic fields and hence can be used to show the local magnetic field configuration. The results of the AS \& E rocket programme over some years has recently been summarized by Vaiana et al. (1974). Their analyses have shown that quite frequently loop structures connect adjacent active regions and trans-equatorial arches are also observed. In general they find that the $\mathrm{X}$-ray emission reaches a maximum above the neutral line of longitudinal magnetic field, and that often, where the field gradients are large, a bright hot small core connects the opposite polarities. The AS \& E observations on Skylab (Vaiana et al., 1973), made with resolution of up to 2 ", will no doubt considerably extend the present information on the spatial distribution of the X-ray emission.

\section{(b) FROM LIMB-TRANSIT OBSERVATIONS}

The spectra and photographs taken of emission on the disk can be supplemented by observations made as an active region rotates beyond the solar limb. Such observations provide useful additional information on the structure as a function of height.

Beigman et al. (1969) used observations made from the Cosmos-166 and Cosmos-230 satellites to show that emission in the wavelength bands $2-8 \AA$ and 8-14 $\AA$, and hence at $T_{\mathrm{e}} \gtrsim 4 \times 10^{6} \mathrm{~K}$ was formed up to $\sim 80000 \mathrm{~km}$ above the limb.

Krieger et al. (1971) made similar studies of the variation of the emission in the range 2.5-12 $\AA$ observed from six active regions with their OSO 4 satellite instrument. They found that the emission $\left(T_{\mathrm{e}}>5 \times 10^{6} \mathrm{~K}\right)$ was formed over heights of from $10^{4}$ to $9 \times 10^{5} \mathrm{~km}$. The large range of height they obtained probably reflects differences in the 


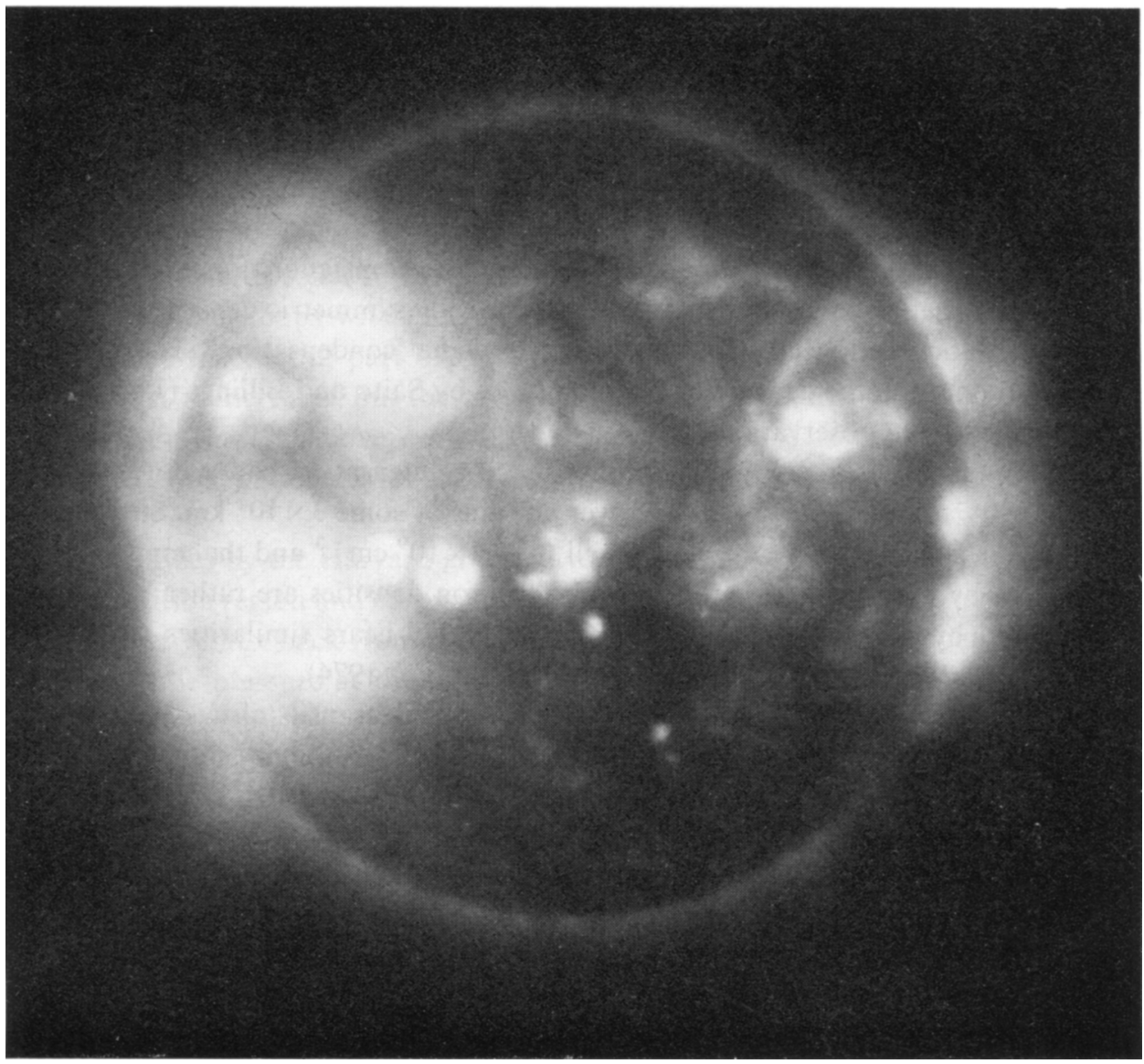

Fig. 9. The X-ray emission from the corona and active regions observed just after the 1970, March 7 eclipse by the AS \& E group (from Van Speybroeck et al., 1970).

temperature structure of the active regions since the height observed by their method will depend on the spectral hardness.

Parkinson (1973) has analyzed flux measurements in the 8.4-9.6 $\AA$ wavelength band, (hence $T_{\mathrm{e}} \simeq 6 \times 10^{6} \mathrm{~K}$ ), obtained from OSO 5, (Herring et al., 1971), and has determined the height of three emitting regions. He finds that the base of the emitting region was $\sim 2 \times 10^{4} \mathrm{~km}$ above the photosphere, and that half the emission is formed below $3 \times 10^{4} \mathrm{~km}$, with some emission extending to $\sim 0.8-1.6 \times 10^{5} \mathrm{~km}$.

The limb-transit observations plus the $\mathrm{X}$-ray images show that in general the hottest material lies at the lowest heights and vice versa, with the emission at $T_{\mathrm{e}} \gtrsim 6 \times 10^{6} \mathrm{~K}$ lying at $\sim 20000 \mathrm{~km}$ above the limb, and that at $T_{\mathrm{e}} \sim 2-3 \times 10^{6} \mathrm{~K}$ extending to $10^{5} \mathrm{~km}$ above the limb. These results are consistent with the spatially resolved EUV data discussed in Section II(c). 


\section{Models Using Combined Observations}

The analysis by Landini and Monsignori Fossi (1971) was discussed in Section II(a), up to the derivation of the emission measure as a function of temperature. These authors and also Parkinson (1973), and Vaiana et al. (1974) have combined X-ray emission measures with models of the geometry in order to find the density and temperature structure of the active regions. Landini and Monsignori Fossi assume equipartition of kinetic and magnetic energy, a spherically symmetric density distribution and hydrostatic equilibrium just at the centre of the 'condensation'. They use as a boundary condition the density distribution given by Saito and Billings (1964), found from visible region observations.

Landini and Monsignori Fossi found that the condensation had a hot core, with $N_{\mathrm{e}} \sim 10^{10} \mathrm{~cm}^{-3}$, and $T_{\mathrm{e}} \sim 3-6 \times 10^{6} \mathrm{~K}$, over a radius of some $3 \times 10^{4} \mathrm{~km}$. Surrounding the core was a region where the density fell to $\sim 2 \times 10^{9} \mathrm{~cm}^{-3}$ and the temperature to $1.5 \times 10^{6} \mathrm{~K}$ by a height of $\sim 10^{5} \mathrm{~km}$. These electron densities are rather higher than those found by other authors, but the overall model bears similarities to those of Boardman and Billings (1969) and Gabriel and Jordan (1974).

Parkinson (1973) has combined the heights from limb transits discussed in Section III(b) with the emission measures found by Batstone et al. (1970). He estimates the emitting volumes at $2-3 \times 10^{6} \mathrm{~K}$ and at $5-6 \times 10^{6} \mathrm{~K}$ as $10^{30} \mathrm{~cm}^{3}$ and $10^{27} \mathrm{~cm}^{3}$, respectively and hence deduces $N_{\mathrm{e}} \sim 2 \times 10^{9} \mathrm{~cm}^{-3}$ at $2-3 \times 10^{6} \mathrm{~K}$ and $N_{\mathrm{e}} \sim 10^{10} \mathrm{~cm}^{3}$ at $T_{\mathrm{e}} \sim 5-6 \times$ $\times 10^{6} \mathrm{~K}$. He suggests an empirical model where the hot material lies at the top of a small loop structure $\left(h \sim 3.0 \times 10^{4} \mathrm{~km}\right)$ below a cooler component extending to $\sim 10^{5} \mathrm{~km}$.

Vaiana et al. (1974) have combined their broad-band photographs with theoretical calculations of the dependence of the emission as a function of wavelength and temperature by Tucker and Koren (1971) and Landini (1974, unpublished). They use two models for the geometry, one an isothermal slab model analogous to an isothermal loop and another which has spherical symmetry. The latter gives the now usual result of low lying regions of hot dense material $\left(T_{\mathrm{e}} \sim 3-3.6 \times 10^{6} \mathrm{~K}, N_{\mathrm{e}} \sim 1.6 \times 10^{10} \mathrm{~cm}^{-3}\right)$, surrounded by an amorphous less dense, cooler region $\left(T_{\mathrm{e}} \sim 2.5 \times 10^{6} \mathrm{~K}, N_{\mathrm{e}} \sim 6 \times\right.$ $\times 10^{9} \mathrm{~cm}^{-3}$ ).

The active region structure found by Aly et al. (1962) from visible region eclipse data is broadly consistent with present results for the temperature region $10^{6} \mathrm{~K}-3 \times$ $\times 10^{6} \mathrm{~K}$, although Aly et al. used a 'condensation' model. Also, the first active region model derived from radio data (Christiansen et al., 1960) gave results similar to those derived with low spatial resolution EUV data.

The exceptionally high resolution $\left(3^{\prime \prime}-9^{\prime \prime}\right)$ radio observations recently reported by Kundu et al. (1974) are particularly interesting. The active region studied appears to be composed of a succession of bright features along the active region. The brightest regions are the central ones where $T_{b} \sim 8 \times 10^{5} \mathrm{~K}$, and regions at lower temperatures $\left(\sim 7 \times 10^{4} \mathrm{~K}\right)$ have a wider separation. It is likely that the radio emission is originating in the dense lower portions of loop structures as seen in lines such as $\mathrm{Si}$ VIII during the March 1970 eclipse. 


\section{The Relation between Observed Structures and Physical Parameters}

There has now been accumulated a considerable quantity of data describing the properties of active regions. It is apparent that the different types of observations are more or less consistent and can be explained by a model that has the following properties; enhanced emission over an area comparable to the plage area, plus loop systems extending outwards some $10^{5} \mathrm{~km}$ above the surface. The X-ray and EUV eclipse data show that larger loops with greater separation of their foot points are cooler than those above the central part of the active region. The density at heights $\sim 10^{4} \mathrm{~km}$ increases as $T_{\mathrm{e}}$ increases, but the variation in $N_{\mathrm{e}}$ is not as large at a given height as is the variation in $T_{\mathrm{e}}$. Above $h \sim 4 \times 10^{4} \mathrm{~km}$ the electron density in the loops which extend to $\sim 10^{5} \mathrm{~km}$ decreases according to hydrostatic equilibrium in an isothermal region.

The analyses by the Harvard group have shown that the emission in active regions from transition region and coronal ions can be understood in terms of variations in $F_{\mathrm{c}}$, the conductive flux back from the corona, in $T_{\mathrm{c}}$, the isothermal coronal temperature, and in $P_{0}$ the pressure at the base of the transition region where $T_{\mathrm{e}} \simeq 2 \times 10^{5} \mathrm{~K}$. In Section II(b) of this paper it was shown how these parameters are inter-related. It appears that the observed properties of active regions at $T_{\mathrm{e}} \geqslant 2 \times 10^{5} \mathrm{~K}$ can be understood in terms of individual elements each with its own $F_{\mathrm{c}}, T_{\mathrm{c}}, P_{0}$ and presumably magnetic field strength. In each flux tube there will be (a) a region where the temperature gradient is given by a constant conductive flux and (b) a region where the temperature is constant at $T_{\mathrm{c}}$. (An intermediate region lies between.) If $T_{\mathrm{c}}$ can be determined then so can $F_{\mathrm{c}}$, but an independent measure of $N_{\mathrm{e}}$ in the constant conductive flux part of the region is needed to specify all the structure. The method is strictly valid only if each flux tube has constant diameter.

The behavior of the spatially integrated emission measure at temperatures $T_{\mathrm{e}}>10^{6} \mathrm{~K}$ can also be understood in terms of adjacent magnetically controlled flux tubes each with its own value of $F_{\mathrm{c}}$ and $P_{0}$. The observed decrease in the total emission measure as a function of temperature is simply produced by the different weightings of each region.

The summation procedure can be expressed as follows:

Let

$$
\int N_{\mathrm{e}}^{2} \frac{\mathrm{d} h}{\mathrm{~d} T} \mathrm{~d} T=Y .
$$

Below $T_{\mathrm{q}}$, the minimum value of $T_{\mathrm{c}}$ (which can be below the temperature of the quiet corona) the emission function at each temperature $T_{n}$ is

$$
\sum_{i} Y_{n}=T_{n}^{b} \sum_{i} a_{i}
$$

where $i$ refers to an individual flux tube, and $T_{i}$ is the maximum temperature in that flux tube. 
Above $T_{\mathbf{q}}$, the total emission at each $T_{n}$ is

$$
\sum_{i} Y_{n}=T_{n}^{b} \sum_{i=n}^{m} a_{i}
$$

where $T_{\mathrm{m}}$ is the maximum temperature present in the whole active region.

At $T_{\mathrm{q}}$, the lowest maximum temperature in any of the flux tubes,

$$
\sum_{i} Y_{\mathrm{q}}=T_{\mathrm{q}}^{3 / 2} \sum_{i=\mathrm{q}}^{m} a_{i}
$$

Then

$$
\sum Y_{n} / \sum Y_{\mathrm{q}}=\left(T_{n}^{3 / 2} / T_{\mathrm{q}}^{3 / 2}\right)\left[1-\left(\sum_{i=\mathrm{q}}^{n} a_{i} / \sum_{i=\mathrm{q}}^{m} a_{i}\right)\right] .
$$

The observed emission function will have a shape determined by $\mathrm{d} \sum_{i} Y_{n} / \mathrm{d} T_{n}$, which in turn depends on how $a_{i}$ varies with $T_{\mathrm{e}}$.

Equation (13) can be compared to the functions that Chambe (1971) and Walker (1972) have used to describe the differential emission measure, i.e. $Y_{n}=Y_{\mathrm{q}} 10^{-T_{n} / T_{q}}$ (Chambe) and

$$
\begin{gathered}
Y_{n}=Y_{0} 10^{-T_{2} / T_{1}}\left(1+1 \cdot 1515 \frac{\left(T_{2}-T_{0}\right)}{T_{1}}-1 \cdot 1515 \frac{\left(T-T_{0}\right)^{2}}{T_{1}\left(T_{2}-T_{0}\right)}\right) \\
T_{0}<T<T_{2} .
\end{gathered}
$$

(Walker)

It can be seen from Equation (13) that it is difficult to go back from the observed total emission function to the physical quantities describing the individual parts of the active region.

Some simplification of (13) can be obtained by expressing $a_{i}$ as a function of $T_{\mathrm{c}}$ only. i.e., let

$$
\left(\begin{array}{c}
a_{i} \\
a_{a}
\end{array}\right)=\left(\frac{T_{\mathrm{c}_{i}}}{T_{a}}\right)^{\chi} \text {, then } \sum_{i} Y_{n} / \sum_{i} Y_{\mathrm{q}}=\left(T_{n}^{3 / 2} / T_{\mathrm{q}}{ }^{3 / 2}\right)\left[1-\left(\sum_{i=\mathrm{q}}^{n} T_{\mathrm{c}_{i}} / \sum_{i=\mathrm{q}}^{m} T_{\mathrm{c}_{i}}\right)\right]
$$

The emission function as a function of $T_{n}$ can then be calculated for different values of $T_{\mathrm{q}}, T_{\mathrm{m}}$ and $\chi$. Figure 10 shows cases with a range of values of $\chi$ and with $T_{\mathrm{m}}=8 \times 10^{6} \mathrm{~K}$ and $T_{\mathrm{q}}=10^{6} \mathrm{~K}$.

(i) $\chi=2.5$

(ii) $\chi=1.0$

(iii) $\chi=0$

(iv) $\chi=-1$

(v) $\chi=-2$

Solutions with lower $T_{\mathrm{m}}$ turn over at lower temperatures, but the overall shapes of the curves are similar. From Figure 10 it can be seen that $1 \leqslant \chi \leqslant-1$ could give a fit to the observations, but it is difficult to determine $\chi$ with higher accuracy.

In Section II, it was found that $\frac{5}{2} \Delta \log T_{\mathrm{c}}=\Delta \log \left(P_{0}^{2} / a\right)$, that $\Delta \log F_{\mathrm{c}}=\Delta \log \left(P_{0}^{2} / a\right)$ and, more importantly, $\Delta \log F_{\mathrm{c}}\left(P_{0}\right)=\frac{5}{2} \Delta \log T_{\mathrm{c}}$. This latter relationship can be compared with Reimers' (1971) conclusion that $\Delta \log F_{\mathrm{c}}=\frac{7}{2} \Delta \log T_{\mathrm{c}}$, which now appears 


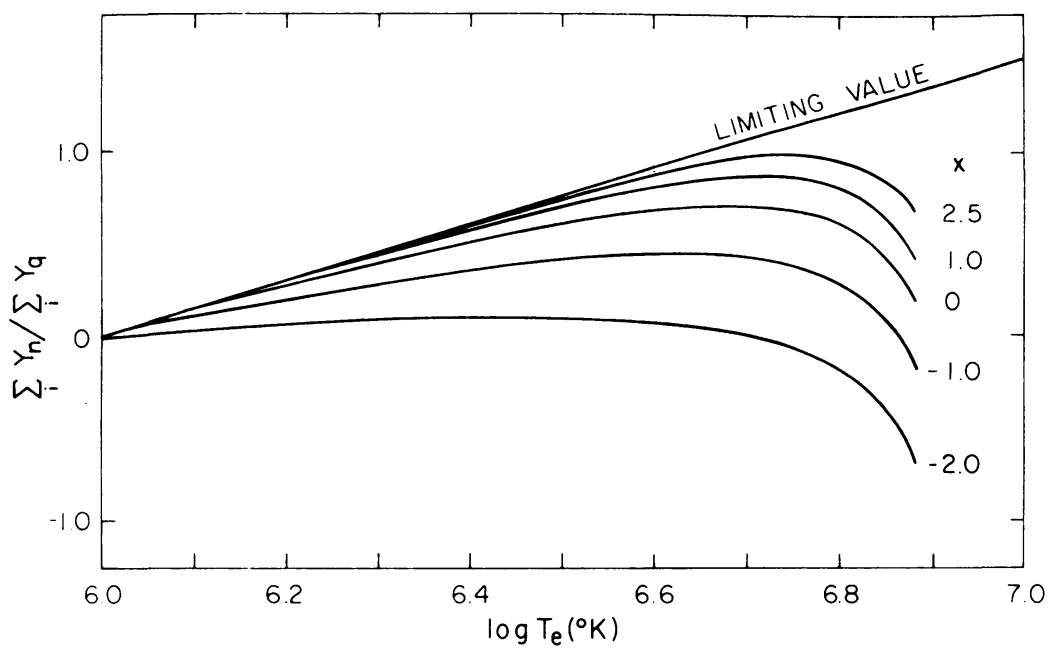

Fig. 10. Theoretical curves for the integrated differential emission measure.

to be incorrect. Reimers derived his relationship between $F_{\mathrm{c}}$ and $T$ as follows; given that,

$$
\begin{aligned}
& F_{\mathrm{c}}=k T_{\mathrm{d} h}^{5 / 2} \mathrm{~d} T \text {, and } F_{\mathrm{c}} \text { is constant, then } \\
& F_{\mathrm{c}}={\underset{7}{7} \frac{2 k\left(T_{\mathrm{c}}^{7 / 2}-T_{0}^{7 / 2}\right)}{h_{\mathrm{c}}-h_{0}}}^{2}
\end{aligned}
$$

The height difference $h_{\mathrm{c}}-h_{0}$ was assumed to be constant, by supposing that $h_{\mathrm{c}}-h_{0} \propto R$, the damping length for waves heating the corona, and hence $\Delta \log F_{\mathrm{c}}=$ $=\frac{7}{2} \Delta \log T_{\mathrm{c}}$. However, $F_{\mathrm{c}}$ is not constant all the way up to $T_{\mathrm{c}}$, but up to some lower value, say $T_{*}$. Thus a relationship $F_{\mathrm{c}}=(2 k / 7)\left(T_{0}^{7 / 2}-T_{*}^{7 / 2}\right) /\left(h_{*}-h_{0}\right)$ would be valid, and since $T_{*}$ can be calculated, may provide a useful means of quickly evaluating height changes, as a function of $F_{\mathrm{c}}$. Reimers also assumed on the basis of earlier work by Unsold $(1960,1970)$ that $N\left(T_{0}\right) \propto F_{\mathrm{m}}$, the mechanical energy flux deposited. Hence $\Delta \log N_{\mathrm{c}}=\frac{5}{2} \Delta \log T_{\mathrm{c}}$. But again the basis of this does not seem to be correct, since $P_{\mathrm{e}}$ is not constant from $P_{0}$ to $P_{\mathrm{c}}$.

Provided spatially resolved observations are available, and it is found that Equation (3) is generally valid, the inter-relations found in Section II(b) and above could be used to determine the distribution of $F_{\mathrm{c}}$ and hence $F_{\mathrm{m}}$ over a given active region. When related to the field strength this should provide useful information on the mechanisms heating the active region material. By substituting a range of values of $F_{\mathrm{c}}$, and $P_{0}$ in the equations it can be seen that the observed properties of the loop systems associated with active regions can be reproduced by a model in which loops emerge from below the photosphere, and expand whilst $F_{\mathrm{m}}$ (and hence $F_{\mathrm{c}}$ ) is decreasing. With a given $F_{\mathrm{m}}$ the temperature at the top of a loop will first increase, as the loop 
expands, to a maximum permitted value, $T_{\mathrm{c}}$, after which an increasing fraction of the loop will become isothermal. These results will be developed further in forthcoming papers.

\section{Acknowledgements}

I wish to thank A. H. Gabriel for allowing me to include results of our analyses of the 1970, March 7 active region prior to their publication elsewhere. It is a pleasure to acknowledge useful discussions with R. S. Peckover.

\section{References}

Acton, L. W., Catura, R. C., Meyerott, A. J., and Wolfson, C. J.: 1972, Solar Phys. $26,183$.

Aly, M., Evans, J. W., and Orrall, F. Q.: 1962, Astrophys. J. 136, 956.

Athay, R. G.: 1966, Astrophys. J. 145, 784.

Batstone, R. M., Evans, K., Parkinson, J. H., and Pounds, K. A.: 1970, Solar Phys. $13,389$.

Beigman, I. L. and Vainshtein, L. A.: 1970, Astron. Zh. 47, 1030.

Beigman, I. L., Grineva, Yu. I., Mandel'shtam, S. L., Vainshtein, L. A., and Žitnik, I. A.: 1969, Solar Phys. 9, 160.

Blake, R. L., Chubb, T. A., Friedman, H., and Unzicker, E.: 1965, Astrophys. J. $142,1$.

Boardman, W. J. and Billings, D. E.: 1969, Astrophys. J. 156, 731.

Bonnelle, C., Senemaud, C., Senemaud, G., Guionnet, M., Henoux, J. C., and Michard, R.: 1973, Solar Phys. 29, 341.

Brabban, D. H. and Glencross, W. M.: 1973, Proc. Roy. Soc. London A334, 231.

Bruzek, A.: 1967, Solar Phys. 2, 451.

Bruzek, A.: 1969, Solar Phys. 8, 29.

Chambe, G.: 1971, Astron. Astrophys. 12, 210.

Christiansen, W. N., Mathewson, D. S., Pawsey, J. L., Smerd, S. F., Boischot, A., Denisse, J. F.,

Simon, P., Kakinuma, T., Dodson-Prince, H., and Firor, J. W.: 1960, Ann. Astrophys. 23, 75.

Dupree, A. K.: 1972, Astrophys. J. 178, 527.

Dupree, A. K., Huber, M. C. E., Noyes, R. W., Parkinson, W. H., Reeves, E. M., and Withbroe, G. L.: 1973, Astrophys. J. 182, 321.

Evans, K. and Pounds, K. A.: 1968, Astrophys. J. 152, 319.

Gabriel, A. H. and Jordan, C.: 1974 (to be published).

Gabriel, A. H., Garton, W. R. S., Goldberg, L., Jones, T. J. L., Jordan, C., Morgan, F. J., Nicholls, R. W., Parkinson, W. H., Paxton, H. J. B., Reeves, E. M., Shenton, D. B., Speer, R. J., and Wilson, R.: 1971, Astrophys. J. 169, 595.

Herring, J. R. H., Glencross, W. M., Parkinson, J. H., and Pounds, K. A.: 1971, Proc. Roy. Soc. London A321, 493.

Howard, R. (ed.): 1971, 'Solar Magnetic Fields', IAU Symp. 43.

Jordan, C.: 1969, Monthly Notices Roy. Astron. Soc. 142, 501.

Jordan, C.: 1971a, in C. de Jager (ed.), Highlights of Astronomy, Vol. 2, D. Reidel Publ. Co., DordrechtHolland, p. 519.

Jordan, C.: 1971b, Solar Phys. 21, 381.

Jordan, C.: 1974, Astron. Astrophys. 34, 69.

Jordan, C. and Wilson, R.: 1971, in C. J. Macris (ed.), Physics of the Solar Corona, D. Reidel Publ. Co., Dordrecht-Holland, p. 219.

Kiepenheuer, K. O. (ed.): 1968, 'Structure and Development of Solar Active Regions', IAU Symp. 35.

Krieger, A. S., Vaiana, G. S., and Van Speybroeck, L. P.: 1971, in R. Howard (ed.), 'Solar Magnetic Fields', IAU Symp. 43, 397.

Kundu, M. R.: 1965, Solar Radio Astronomy, John Wiley-Interscience Publ.

Kundu, M. R., Becker, R. H., and Velusamy, T.: 1974, Solar Phys. 34, 185.

Landini, M.: 1974 (unpublished).

Landini, M. and Monsignori Fossi, B. C.: 1971, Solar Phys. 17, 379.

Livingston, W., Harvey, J., and Slaughter, C.: 1970, Nature 226, 1146.

Munro, R. H., Dupree, A. K., and Withbroe, G. L.: 1971, Solar Phys. 19, 347. 
Noyes, R. W.: 1971, in C. J. Macris (ed.), Physics of the Solar Corona, D. Reidel Publ. Co., Dordrecht-Holland, p. 192.

Noyes, R. W., Withbroe, G. L., and Kirshner, R. P. : 1970, Solar Phys. 11, 388.

Parkinson, J. H.: 1973, Solar Phys. 28, 487.

Pottasch, S. R.: 1963, Astrophys. J. 134, 347.

Pottasch, S. R.: 1967, Bull. Astron. Soc. Neth. 19, 113.

Reimers, C.: 1971, Astron. Astrophys. 10, 182.

Saito, K. and Billings, D. E.: 1964, Astrophys. J. 140, 760.

Speer, R. J., Garton, W. R. S., Goldberg, L., Parkinson, W. H., Reeves, E. M., Morgan, J. F., Nicholls, R. W., Jones, T. J. L., Paxton, H. J. B., Shenton, D. B., and Wilson, R.: 1970, Nature 226, 249.

Tandberg-Hanssen, E.: 1967, Solar Activity, Blaisdell Publ. Co., Waltham, Mass.

Tousey, R.: 1967, Astrophys. J. 149, 239.

Tucker, W. H. and Koren, M.: 1971, Astrophys. J. 168, 283.

Underwood, J. H. and Muney, W. S.: 1967, Solar Phys. 1, 129.

Unsold, A.: 1960, Z. Astrophys. 50, 57.

Unsold, A.: 1970, Astron. Astrophys. 4, 220.

Vaiana, G. S., Davis, J. M., Giacconi, R., Krieger, A. S., Silk, J. K., Timothy, A. F., and Zombeck, M.: 1973, Astrophys. J. 185, L47.

Vaiana, G. S., Krieger, A. S., and Timothy, A. F.: 1974, Solar Phys. (in press).

Van Speybroeck, L. P., Krieger, A. S., and Vaiana, G. S. : 1970, Nature 227, 818.

Walker, A. B. C., Jr.: 1972, Space Sci. Rev. 13, 672.

Walker, A. B. C., Jr. and Rugge, H. R.: 1968, Astron. J. 73, S81.

Walker, A. B. C., Jr., Rugge, H. R., and Weiss, K.: 1973, Aerospace Report No TR-0074 (9260-02)-2.

Wiese, W. L., Smith, M. W., and Glennon, B. M.: 1966, NSROS-NBS4 (U.S. Govt. Printing Office, Washington, D.C.).

Wiese, W. L., Smith, M. W., and Miles, B. M.: 1969, NSROS-NBS22 (U.S. Govt. Printing Office, Washington, D.C.).

Withbroe, G. L.: 1970, Solar Phys. 11, 42.

Zirin, H.: 1966, The Solar Atmosphere, Blaisdell Publ. Co., Waltham, Mass. 